\title{
Wir werden uns wiederfinden - didaktische Anregungen zur kreativen Behandlung des Themas Vertreibung
}

\section{Milena Kubátová}

This article deals with the topic of displacement from Sudetenland in the German children and juvenile literature. Based on the book Wir werden uns wiederfinden by Annelies Schwarz, this theme will be presented through project-oriented teaching and creative writing activities. Political facts are intentionally not described. While interpreting the book, I focus on the emotional tension and the human side of the events which is depicted or missed out by the book. While dealing with the text, the attention is focused on creativity and empathy.

Sudetenland and displacement - children and juvenile literature - Annelies Schwarz project-oriented teaching - creative writing

Dieser Artikel befasst sich mit dem Thema „Vertreibung aus dem Sudetenland“ in der deutschsprachigen Kinder- und Jugendliteratur. Anhand des Buches Wir werden uns wiederfinden von Annelies Schwarz wurde diese Thematik einer Gruppe von Deutschlernern an der Universität mit einigen Aktivitäten des handlungs- und produktionsorientierten Unterrichts im Zusammenhang mit dem kreativen Schreiben präsentiert. Mit Absicht wurden aber die politischen Fakten nicht gestreift. In den Unterrichtseinheiten wurde vielmehr auf die emotionale Seite der menschlichen Handlungen, die in dem ausgewählten Buch dargestellt oder auch ausgelassen wurden, Wert gelegt. Bei einem solchen Umgang mit einem literarischen Text ist die gesamte Aufmerksamkeit auf die Kreativität und auf die Stärkung der Empathie gerichtet.

Sudetenland und Vertreibung - Kinder- und Jugendliteratur - Annelies Schwarz - handlungsund produktionsorientierter Unterricht - kreatives Schreiben

\section{Einleitung}

In dem vorliegenden Beitrag wird das Ziel verfolgt, anhand einiger Aktivitäten darzustellen, wie man sich mit einem literarischen Text im DaF-Unterricht kreativ, unterhaltsam und spielerisch, vor allem aber auch lernzielorientiert auseinandersetzen kann. Als Grundlage für diese Studie wurde der Jugendroman Wir werden uns wiederfinden ${ }^{I}$ von Annelies Schwarz ausgewählt, der die Vertreibung aus dem Sudetenland thematisiert. Die Leitidee der Unterrichtsgestaltung war nicht nur die eigentliche Textarbeit, Ziel war es auch, die gesamte Persönlichkeit der Lernenden auf der kreativen und emotionalen Ebene anzusprechen. Aus diesem Grund befasst sich das Konzept mit der Frage, welche Emotionen dieses Buch beinhaltet und wie man die Gefühle kreativ in die Textanalyse einbeziehen könnte.

1 Dieser Roman wurde von K. Kaiserová und H. Tomková ins Tschechische übersetzt und 2003 als Červenobilé křeminky: přiběh Liese 1944-1950 in Ústí nad Labem veröffentlicht. 
Die Arbeit beschäftigt sich mit den emotionalen Spannungen und mit den inneren Befindlichkeiten der Menschen in Bezug auf die Vertreibung sowie deren Darstellungen bzw. Auslassungen im Text. Das ausgewählte Buch bietet natürlich Wissenszusammenhänge aus dem Bereich der deutschsprachigen Kultur, Landeskunde und Geschichte, sowie sprachlichen Stoff sowohl in lexikalischer als auch grammatischer Hinsicht, die man in den Unterrichtseinheiten sehr wohl besprechen könnte, sie werden hier aber absichtlich außer Acht gelassen.

Der Schwerpunkt dieser Arbeit liegt auf der kreativen Behandlung der Emotionen in Bezug auf den handlungs- und produktionsorientierten Unterricht. In diesem Zusammenhang standen vor allem die praktischen Fertigkeiten, die man den affektiven und psychomotorischen Zielen zuordnet, im Vordergrund. Die einzelnen Lernziele werden bei jeder Unterrichtseinheit näher spezifiziert. Die Auseinandersetzung mit den Schrecken der Kriegszeit kann unter die Haut gehen, deswegen muss man beim Unterricht darauf achten, dass die Lerner freiwillig an den Aktivitäten teilnehmen. Am Anfang liegt der Schwerpunkt auf nicht zu anspruchsvollen Tätigkeiten, um emotionale Barrieren und eine meist vorhandene Anfangsschüchternheit zu überwinden. Bei der Auswahl der Arbeitsaufgaben wurde angestrebt, die Selbständigkeit, die emotionale Wechselwirkung und den Schwierigkeitsgrad der Übungen langsam zu steigern. Elemente des handlungs- und produktionsorientierten Unterrichts sollten auf allen Ebenen vertreten sein.

\section{Forschungsgebiet}

Im Vordergrund der Textanalyse stand die Problematik der Vertreibung auf der emotionalen Ebene, ihre Darstellung im ausgewählten Buch und eine mögliche Behandlung im Deutschunterricht. Das Buch wurde im Rahmen eines Projektes an der Philosophischen Fakultät der Masaryk-Universität behandelt, an dem Studenten auf unterschiedlichem Sprachniveau teilnahmen. Bei der Realisierung wurde kein geschichtliches Wissen vermittelt, ${ }^{1}$ sondern Ziel war es, die Studenten mit Texten und Bildern aus dem ausgewählten Buch zu konfrontieren und ihre Gefühle und Assoziationen wirken zu lassen.

Dieser Aspekt der Textanalyse verlangte von den Studenten nicht nur einfaches Lesen, sondern auch das Umgestalten und das Ergänzen von Textabschnitten. Die Texte wurden kreativ (szenisch, bildlich und akustisch) weiterverarbeitet. Während der Untersuchung wurde das Augenmerk vor allem auf die literarischen, emotiven und kreativen Kompetenzen gerichtet, wie sie Gerhard Haas (Haas 2001: 34-37) in Bezug auf den Literaturunterricht präsentiert. Darunter versteht man die verschiedenartigen Fähigkeiten, die für das Umgehen mit einer Lektüre und ihr Verstehen erforderlich sind.

1 Es wurde von dem Vorwissen der Studenten aus den auf Geschichte orientierten Seminaren ausgegangen. 


\subsection{Thematischer Einstieg}

Lernziele: Bekanntmachung mit dem Thema, mit dem Buch, mit der Autorin, Gedächtnisübung

Während der ersten Unterrichtseinheit wurden die Studenten in kleinen Schritten mit der Thematik des Buches bekanntgemacht. Damit wurde ihr Interesse geweckt. Die erste Stunde eignet sich dazu, das Thema der Vertreibung aus dem Sudetenland anzusprechen, um es danach näher zu bearbeiten.

Begonnen wurde im Plenum mit der Präsentation des einzigen Bildes auf dem Umschlag der ausgewählten Lektüre, wobei von den Studenten erste Vermutungen zu dem noch unbekannten Buch geäußert wurden. Danach wurde die Autorin anhand einiger Fotos vorgestellt. Im Folgenden wurde näher auf den Prätext eingegangen und die das Buch beschreibenden Informationen wurden schrittweise an die Tafel geschrieben: Name der Autorin (Annelies Schwarz), Titel des Buches (Wir werden uns wiederfinden) und Untertitel (Die Vertreibung einer Familie). Bei jedem neuen Hinweis kamen unterschiedliche Äußerungen von den Studenten und das Thema wurde näher konkretisiert. Die Arbeit verlief in zwei Gruppen und aus den in den Gruppen gesammelten Gedanken wurden zwei zum Thema passende Wörter abgeleitet: Hoffnung und Sudeten.

Zum Abschluss wurde kurz mit dem Inhalt gearbeitet. Während dieser Aktivität wurde den Studenten die Inhaltsangabe geschildert, sie mussten ihr Gedächtnis üben und dabei wurden sie mehrmals mit Aussagen wie „aus der Heimat vertrieben“, „Flüchtlinge aus Schlesien“, „überfüllte Viehwaggons und Lager“, „Flucht und Vertreibung", „Gefühle“, „Angst“, „menschliche Beziehungen“ oder „Heimweh" konfrontiert. Bei der mündlichen Weitergabe der Inhaltsangabe ${ }^{1}$ kam es erwartungsgemäß zu Informationsverlust, was schließlich zu folgendem Ergebnis führte: Die Autorin schildert eine traurige, aber sehr interessante Geschichte, die sich in einem Zug abspielt. Eine Großfamilie wollte weg aus Schlesien, sie konnte aber nicht. Die Hauptfigur, ein Mädchen, starb unglücklicher Weise, weil sie aus einem Zug rausgesprungen ist.

\subsection{Deutsch-tschechische Beziehungen}

Lernziele: Orientierendes Lesen, Leseverstehen, visuelle Gestaltung der Textstellen, Verfassen eines Gedichtes und eines Textes

1 Fünf Personen warteten vor der Tür, sie sollten einzeln nacheinander in das Klassenzimmer kommen und jeweils die Inhaltsangabe weiter nacherzählen, die sie von der Kollegin davor gehört hatten. Die anderen Studenten folgten den Aussagen, die weitergegeben wurden, notierten die Informationen und stellten fest, wie sich der Inhalt durch die mündliche Weitergabe verändert hatte. 
Zu Beginn der zweiten Stunde wurden die wichtigsten Fakten an der Tafel gesammelt: Autorin, Zeit und Ort, Hauptfigur, deren Familie, weitere wichtige Figuren.

Die Studenten sollten vorher zu Hause die ersten ausgewählten Kapitel (Schwarz 1988: 5-6, 17-29) lesen und bekamen nun die Gelegenheit, sich über das Buch frei zu äußern und die emotionale Wirkung der Lektüre zu beschreiben.

Im weiteren Verlauf der Unterrichtseinheit wurden die Studenten in drei Gruppen eingeteilt. Jede Gruppe wurde aufgefordert, thematisch verschiedene Textstellen (s.u. a bis c)

a) Krieg mit den Augen eines Kindes (Ungewissheit und Zweifel)

b) Stellung der Erwachsenen (Ärger und Besorgnis)

c) wesentliche Merkmale des Krieges (Aggression und Arroganz)

in der Lektüre herauszufinden und danach die wichtigsten Aussagen und Gefühle aus dem Buch visuell zu gestalten, d. h. als Bild zu präsentieren, als Bildcollage des Textes zu erarbeiten oder Schreibgestaltung als Interpretationsform zu wählen.

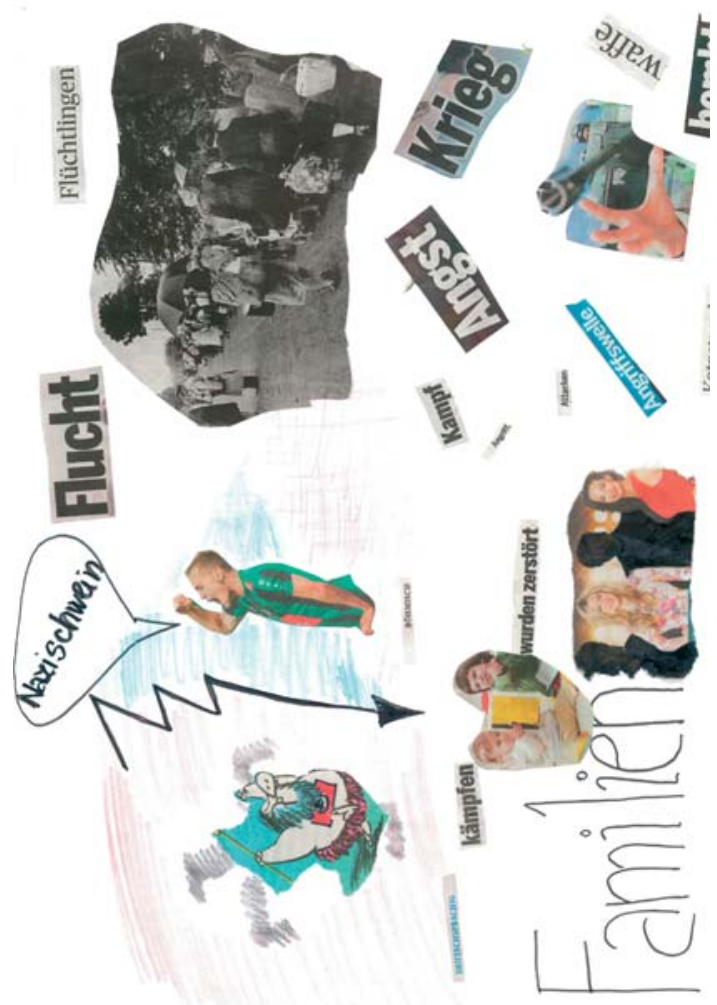

Abb. 1: Eine der entstandenen Bildcollagen 
Beim nächsten Gespräch folgten Hinweise auf die Flüchtlinge (Schwarz 1988: 27-29) und es wurde die Frage gestellt, wie es jemandem ergeht, der seine Heimat verlassen muss. In diesem Zusammenhang wurden die Studenten in zwei Gruppen eingeteilt und gebeten, zehn passende Wörter zu zwei Themen zu finden. Diese Wörter waren:

a) Thema „Zu Hause bleiben (können)“: Sicherheit, Heimat, Geborgenheit, Freundschaft, Erinnerungen, Gemütlichkeit, Erleichterung, zufrieden sein, kein Stress, sorglos sein;

b) Thema „Heimat verlassen (müssen)“: Hoffnung, Freudenverlust, Trennung, Angst, Rettung, neuer Anfang, neue Sprache, Schwierigkeiten, Unsicherheit, bessere Zukunft?

Die Gruppen tauschten danach die Schlüsselwörter aus und erhielten die Hausaufgabe einen kurzen Prosatext zum Thema a) mit den Wörtern von Thema b) und umgekehrt zu verfassen.

Anschließend wurde das nächste aus dem Buch entnommene Thema „Tschechen sind Freunde, aber gleichzeitig Feinde" auf kreative Art und Weise behandelt. Den Studenten wurden schriftlich elf Entscheidungsfragen gestellt, die sich auf den emotionalen Sachverhalt in dem Textabschnitt (Schwarz 1988: 33-34, 38-40) bezogen. Im Rahmen der Antwort (ja - nein, bzw. richtig - falsch) wurden zwei verschiedene Buchstaben zur Auswahl gestellt. Falls die Fragen in Bezug auf den Text richtig beantwortet und die Buchstaben in der richtigen Reihenfolge aufschrieben wurden, ergab sich der mit Gefühlen verbundene Ausdruck HERZKLOPFEN. Die Studenten sollten zu diesem Wort ein reimloses Akrostichon ${ }^{1}$ schreiben, das ihre eigenen Ängste beschreibt und zum Ausdruck bringt. Wie kreativ die Studenten diese Aufgabe erledigten, wird hier mit diesen beiden Beispielen demonstriert ${ }^{2}$ :

Hören kann ich es nicht,

Empfinden doch.

$\boldsymbol{R} u$ he verlässt meinen Leib,

Zittern dringt in ihn ein.

Klare Sinne werden betäubt,

Laune verdorben.

Obwohl ich bange bin,

Probier' ich Mut zu fassen.

Finger der Ängste sind jedoch zu lang,

Endlos wollen sie mich in Gefangenschaft halten.

Nie wieder will ich so was erleben
Hier und da.

$\boldsymbol{E}$ s sind überall.

Riechen nach dem Tod.

Zeigen sich in meinen Träumen.

Klopfen an die Tür.

Lauern auf mich.

Opfer soll ich sein?

Plötzlich sind alle weg:

Freunde, Familie...

Einsam soll ich sein?

Nein!

1 Das Akrostichon ist eine Textform, in diesem Fall ein Gedicht, bei der die Anfangsbuchstaben eines Wortes senkrecht untereinander geschrieben werden. Jeder dieser Buchstabe bildet dann den Anfang einer neuen Aussage.

2 Die Gedichte wurden bewusst nicht korrigiert. 


\subsection{Von Zuhause vertrieben}

Lernziele: Kommunikationsfähigkeit - Rollenspiel, Gestaltung der Spielszene, Verfassen eines inneren Monologes einer Figur, emotionales Streitgespräch

Die dritte Stunde wurde direkt mit der folgenden Leseprobe eröffnet:

Mutter brachte ein weinendes Mädchen zu mir: „Du, Liese, sieh nur, wie traurig es ist, es musste heute so schnell aus seinem Haus, dass es seine Puppe nicht mehr mitnehmen konnte. Bringst du ihm eine von dir?"

[...] Ich nahm die Minka mit den schwarzen Zöpfen, ich mochte sie am liebsten. Als ich sie dem Mädchen geben wollte, fing es wieder an zu weinen. Seine Mutter sagte: „Weißt, ihre Lotti hatte blonde Zöpfe, aber sie wird sich schon an deine Puppe gewöhnen."

"Sie muss aber meine Minka liebhaben, sonst geb ich sie nicht her", sagte ich, und die Mutter erwiderte: „Wird sie schon, aber jetzt müssen wir schnell zum Wagen, sie rufen schon nach uns."

[...] Der setzte sich schon in Bewegung, als sie noch hinaufkletterten. Ich sah noch, wie das Mädchen oben Minka in die Hand nahm.

Meine Minka fuhr fort. (Schwarz 1988: 59)

Nachdem diese ungekürzte Textstelle gemeinsam laut und in verteilten Rollen (Erzähler - innere Stimme, Liese, ihre Mutter, ein Mädchen, Mutter des Mädchens, Polizist und Kutscher) vorgelesen wurde, sollten die Studenten die Situation in zwei Gruppen in einem lebenden Bild (als Foto dieser Spielszene) wiedergeben. Nach Überwindung der ersten Schüchternheit arbeiteten alle sehr gut mit, die Szenen wurden durch emotionale Ausdrücke (Weinen, Ausrufe) unterstützt und man konnte die Emotionen auch an Mimik und Gestik ablesen, was erwünscht war.

Liese hatte dem fremden Mädchen ihre Lieblingspuppe gegeben, obwohl dieses von dem Geschenk gar nicht so begeistert war. Die Szene bot Gelegenheit, einen inneren Monolog darüber zu schreiben, was Liese empfunden und woran sie gedacht hat. Die Studenten sollten sich vorstellen, sie seien Liese und mit den Worten „Meine Minka fuhr fort“ einen Text beginnen. Diese Übung verlangte, sich unter Zeitdruck in die Situation einzufühlen. Dadurch konnte die Gefühlswelt eines kleinen Mädchens verdeutlicht werden. Obwohl den Studenten für die Bearbeitung dieser Aufgabe nur fünf Minuten zur Verfügung gestellt wurden, entstanden gelungene Texte, beispielsweise:

„Meine Minka fuhr fort... Nur ibre braune Haare und weißes Kleid sehe ich in der Ferne. Meine Lieblingspuppe, meine Freundin, der ich mich mit allem vertrauen konnte. Und jetzt hat sie das fremde Mädchen. Das Mädchen mit den schmutzigen, dicken Fingern. Was wird mit Minka?" 
Als Übergang zur nächsten Aktivität wurden im Plenum die zu Hause geschriebenen Texte besprochen und analysiert. Dabei wurden den Schreibenden auch Fragen gestellt: Wo und wann spielt sich die Geschichte ab? Mit welchen Problemen haben Sie oder mussten Sie beim Schreiben kämpfen? Worauf haben Sie sich bei Ihrem eigenen Text konzentriert - auf die Emotionen oder auf die Tatsachen? Wie ist es Ihnen gelungen, sich mit den Themen auseinanderzusetzen? Stellvertretend für diese Texte ist unten ein Text zum Thema „Heimat verlassen (müssen)“ abgedruckt. Der Text wurde bewusst nicht korrigiert:

Den Abend, habe ich wieder nicht einschlafen können. Ich wusste, dass schon Morgen der Tag kommt, in dem sich alles endern soll.

Wird es woll eine Erleichterung sein? Vieleicht...

Vieleicht werde ich keinen Stress mehr fühlen. Wer weiss...

Bleibt die Freundschaft, die ich heir endlich gefunden habe, oder verstaltet es sich nur in Erinnerungen?

Kann ich so zufrieden werden?

Mutter sagt, dass wir uns bald geborgen fühlen werden. Aber woher hat sie diese Sicherheit?

Auf einer Seite kann es sorgloser sein, wir werden dort ja von niemanden verfolgt. Aber auf der anderen Seite...

Kann mir Jemand meine Fragen beantworten?

Ich bin unsicher...

Die Gemütlichkeit wird verschwinden...

Den meine Heimat, die bleibt hier...

Das Verhalten der deutschen Soldaten und der Nationalsozialisten gehört zu den Themen, die in dem Buch nicht behandelt werden. An dieser Stelle wurde nicht angestrebt, die geschichtlichen Ereignisse zu bearbeiten, sondern es ging darum, mit der Darstellung der starken Emotionen die Studierenden zu konfrontieren. $\mathrm{Zu}$ dem oben erwähnten Thema wurde eine passende Diskussion (ein Rollenspiel) in Form einer Gerichtsverhandlung gestaltet, wobei der Lehrer als Richter mit der Anklage hervortrat. Die Studenten wurden in zwei Gruppen eingeteilt. Eine Gruppe übernahm die Rolle des Angeklagten (die Deutschen), die andere die Rolle des Verteidigers (die Tschechen), in denen sie bei Gericht auftreten sollten. Den Lernenden in der Gruppe wurde dabei Zeit zur Vorbereitung verschiedener Argumente gegeben. Danach sollten sie wie Juristen handeln: höflich, aber energisch sprechen, mit Argumenten überzeugen, die eigenen Interessen verteidigen, Zwischenfragen stellen, Aussagen bezweifeln usw. Bei dieser Übung wurde die Arbeitsatmosphäre bei Gericht sehr wirksam und überzeugend dargestellt.

Weil man bei dieser Übung vom Buch ausging, stand das Urteil nach dem Prozess eindeutig fest und lautete: „Lieses Familie wird morgen um 10 Uhr ausgewiesen. Jeder Deutsche kann höchsten zwanzig Kilo Reisegepäck haben. Es ist nicht erlaubt, wertvolle Sachen und Tiere mitzunehmen." 


\subsection{Emotional angespannte Atmosphäre}

Lernziele: Beenden eines Textes und seine szenische Darstellung, Wahrnehmung des Textes mit anderen Sinnen

Als Einstieg in die Stunde wurde mit den Studenten die vom Lehrer gekürzte Textstelle (Schwarz: 67-68), in der die Familie das Haus verlässt, vorgelesen.

Der belastende Weg in ein fremdes Land begann in Arnau (Hostinné), wo es zu einem Konflikt zwischen einem tschechischen Soldaten und der Großmutter kam. Als sie in der Nacht auf die Toilette musste, kam sie ohne ihren Mantel mit diesen Worten zurück:

„Stellt euch so was vor“, sprach sie ganz aufgeregt, „der Wachtposten hat ihn mir einfach heruntergezogen und gesagt: Den brauchst du jetzt nicht mehr.“ (Schwarz 1988: 72)

Am nächsten Tag versuchte die Mutter mit dem Soldaten zu reden. Die Aufgabe der Studenten war es, diesen im Buch fehlenden Dialog zu spielen. In diesem Gespräch, das vorher nicht vorbereitet wurde, durften sie nur die Wörter BITTE und NEIN verwenden und sie mussten auf ihren Spielpartner sofort reagieren. Es war erstaunlich, wie unterschiedlich die Lösungen bei den kleinen Auftritten, die aufgeführt wurden, waren. Es zeigte sich sehr gut, wie sich die Studenten in die Situation einfühlen und die Problematik wahrnehmen konnten.

Nachdem alle Partnerpaare ihre Szenen dargestellt hatten, wurde der Gruppe die strafende Konsequenz aus dem Buch vorgelesen, die Lieses Mutter für ihr Bemühen und ihren Mut tragen musste:

„Und du gib deinen auch her", war seine Antwort, während er Mutter festhielt und ihr auch noch den ihren auszog. Wir sahen es mit an und konnten nichts dagegen tun. Mit Mutters Mantel, der innen mit Pelz gefüttert war, ging der Soldat weg, und Mutter blieb nichts anderes übrig, als schnell zu uns zurückzukehren [...]. (Schwarz 1988: 74)

Auf den weiteren zum Lesen vorgelegten Seiten (Schwarz 1988: 75-80) beschreibt die Autorin die emotional angespannte Atmosphäre auf der langen Fahrt nach Deutschland, die Fahrt mit einem Güterzug, wo sonst nur Vieh transportiert wird, das ewige Warten auf den Bahnhöfen, den Aufenthalt in den überfüllten Waggons und Lagern. Im nächsten Schritt sollten sich die Studenten durch eine Phantasiereise in diesen Weg einfühlen. Sie sollten sich vorstellen, sie wären Liese und unternehmen diese Reise im Güterzug. Zu Hause sollten sie sich Gedanken darüber machen, wie man die Erlebnisse mit höchstens zwei der fünf Sinnen wahrnehmen und reflektieren könnte. Es wurde erwähnt, dass der Text mit akustischen Mitteln ${ }^{1}$ (z. B. mit Orff-

1 Die Studenten hatten zur Auswahl Glockenspiel, Beckenpaar, Rasselei, Klanghölzer, Glockenkranz, Rumba-Kugeln, Schellenring/Trommel, Triangel. Zur Gestaltung passender Hintergrundmusik 
Instrumenten) vertont oder mit eigenen (selbst gemalten) Bildern begleitet werden konnte. Die entstandenen Arbeitsergebnisse hatten einen künstlerischen Wert, den ich an dieser Stelle leider nicht wiedergeben kann. Die Studenten komponierten ein eigenes Musikstück, das zu Hause aufgenommen und mit Bildern unterlegt wurde. Die Zugsreise wurde akustisch mit einer Geige dargestellt, außerdem wurde sie durch gespieltes Gedränge und mit verbundenen Augen direkt durchlebt. Bei diesen Übungen wurden die Emotionen am höchsten angerührt.

\subsection{In Deutschland angekommen}

Lernziele: Kommunikationsfähigkeit, Leseverstehen, Comics-Darstellung, Beenden und szenische Darstellung des Textes

Im Folgenden wurde das Thema „Hilfe“ bearbeitet. Es wurden drei Textpassagen (Schwarz 1988: 85-90) ausgesucht, in denen das Verhalten der Personen positiv dargestellt wurde, obwohl man beim Lesen der ersten Zeilen zuerst anderer Meinung sein könnte. Die Studenten wurden in drei Gruppen eingeteilt, wobei sich jede Gruppe mit einem anderen Textabschnitt befasste. Nach dem Lesen setzte sich jeweils ein Freiwilliger auf den sogenannten „Heißen Stuhl“ und stellte eine Figur aus dem Text dar. In der übernommenen Rolle versuchte er, die Fragen des Lehrers bzw. der anderen Studenten zu beantworten, gleichzeitig aber auch sein Verhalten zu verteidigen.

a) zwei Frauen mit großen, grauen Umhängetaschen, auf denen ein rotes Kreuz zu erkennen war; sie sprachen mit den Menschen, manchmal gaben sie den Angekommenen kleine Zettel (Essensbons), manchmal nicht;

b) eine Frau im Garten, hat der Familie den Rücken zugedreht; nach einer Weile und aufgrund Wolfgangs (der kleine Bruder) Weinens reichte sie Lieses Mutter wortlos eine Handvoll Möhren;

c) ein Mann, der vom Lastwagen Möhren und Kohlrabis verkaufte; er wollte vor den anderen Menschen nichts umsonst geben, trotzdem warf er ab und zu ein paar „schlechte“ Möhren, die gar nicht schlecht waren, zu Boden und in Richtung der Familie.

Einige Studenten gaben zu, dass es für sie sehr schwierig und unangenehm war, das Verhalten der Figuren zu verteidigen.

Zum Schluss des Projektes beschäftigten sich die Studenten nacheinander mit drei emotional berührenden Texten, die das Ankommen nach Deutschland schildern.

eignen sich auch andere Gegenstände wie z. B. Töpfe und Löffel, Kamm, Papier. Man sollte dabei aber keineswegs vergessen, dass man einen Text durch die eigene Stimme und verschiedene Vortragsweisen beeinflussen kann. 
Es war wieder ein Zug, der sonst zum Viehtransport benutzt wird. Aber wir kannten das schon und waren nicht weiter verwundert. [...] Am Morgen weckte mich lautes Stimmgewirr. „Haltet doch das Mädel, sie fällt!“ Ein Aufprall, Rufe. [...] Mutter und einige Leute waren sofort draußen, standen und knieten um sie herum. „Der Puls geht schwach“, sagte Mutter besorgt. „Ich gehe zur Bahnhofswache“, sagte ein alter Mann und verschwand zwischen den Leuten. (Schwarz 1988: 91-92)

In dieser Leseprobe hatte die Mutter große Sorgen. Den Studenten wurde eine ganz logische Frage gestellt: Was für ein Mädchen lag auf dem Boden? Was ist passiert? Wie wird die Familie auf die entstandene Situation reagieren? Gelingt es ihnen, eine Lösung zu finden? Danach sollten die Studenten in Zweiergruppen die ausgelassene Vorgeschichte und die Fortsetzung selber entwerfen. Die Studenten wurden um gründliches Lesen gebeten und sie wurden an ihr Vorwissen erinnert. Alle stellten richtig fest, dass hier nicht die Hauptprotagonistin in Schwierigkeiten geraten war, da im Text steht, dass die Menschen „um sie herum“ knieten, das Buch aber in der Ich-Form geschrieben ist. Hauptsächlich wurde ein fremdes Mädchen, die Schwester von Liese oder ihre Freundin erwähnt. Niemand hatte an die Haushelferin Marienchen gedacht.

Die zweite Leseprobe (Schwarz 1988: 93) bezog sich auf die Emotionen, die im Text nicht nur angedeutet, sondern auch explizit beschrieben werden (Enttäuschung, Sehnsucht, Tränen, Erleichterung, Zorn, Ärger). Nun wurden die Studenten aufgefordert, wieder in Gruppen die Textstelle in Bildern als Comics darzustellen und sich darauf zu konzentrieren, was Lieses Gefühle sagen würden, wenn sie sprechen könnten. Die folgende Illustration soll zeigen, wie die Emotionen zeichnerisch umgesetzt wurden.

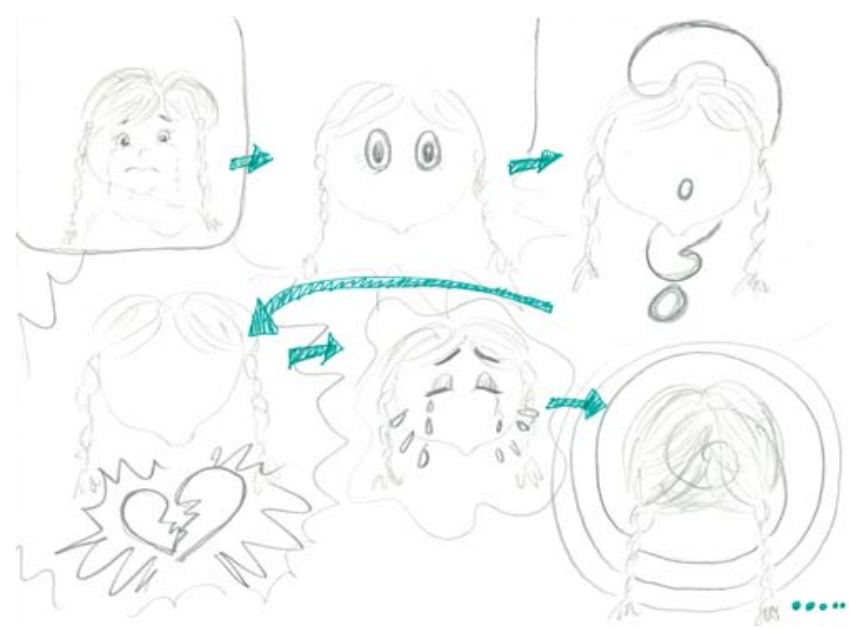

Abb. 2: Lieses Gefühle 
Mit dem dritten Textabschnitt wurden die Studenten sowohl mit den Emotionen als auch mit dem Dialekt konfrontiert:

Einmal hörte ich, wie ein Mädchen zu ihrer Mutter in die Küche hineinrief: "Mutter, krieg ich'ne Bemme?" Nach einer Weile reichte ihr eine Hand [...]. Ich fasste mir ein Herz und sagte zu dem Mädchen: „Du, kann ich auch eine kriegen?“ Das Mädchen rief wieder: „Gibst du dem Flichtling auch eine Bemme?" Und von drinnen ... (Schwarz 1988: 100)

Das Wort „Flichtling “ war verständlich, aber was wollte das Mädchen von der Mutter? Und wie hat die Mutter reagiert? Das herauszufinden waren die Arbeitsaufträge. Die Studenten sollten in drei Gruppen Überlegungen anstellen, was „eine Bemme“ sein könnte, wie man das Gespräch mit weiteren Aussagen und mit einer Antwort beenden könnte. Erst als alle drei Gruppen ihre Dialoge in Szenen dargestellt hatten, wurde den Studenten die Originalfassung zur Verfügung gestellt.

[...] von drinnen kam es: „Nee, für die Flichtlinge haben wa geene.“ Das Mädchen sah mich an und schüttelte den Kopf. Dann brach sie mir von ihrer Bemme ein Stück ab, und ich teilte das Stück nochmal mit Christl. Wir kauten alle drei und lachten uns an. (Schwarz 1988: 100)

Überraschend war, dass eine Gruppe das Gespräch sehr ähnlich dem der Autorin beendete. Die Fehler wurden wieder bewusst nicht korrigiert:

Mutter: Welchem Flüchtling? Ich habe nur wenig davon.

Liese: $\quad$ Und warum? Du bist ein Mädchen wie ich.

Mädchen: Und warum? Sie ist ein Mädchen wie ich.

Mutter: $\quad$ Tut mir Leid, es ist nur für unsere Familie.

Liese: [weinen]

Mädchen: $\quad \mathrm{Na}$, guck mal, ich habe es nicht ganz gegessen, ich gebe dir einen Teil.

Liese: $\quad$ Aber ... wirst du nicht hungrig?

Mädchen: Nein, mir reicht es, ich habe genug.

Liese: $\quad$ Wirklich? Danke! Wir werden Freundinnen!!! :-)

\section{Zusammenfassung}

In dem vorliegenden Artikel wurde die mit dem Thema Vertreibung verbundene Lektüre von Annelies Schwarz vorgestellt und die psychischen Belastungsfaktoren dieser Problematik bearbeitet. Anhand einiger Textstellen aus dem Buch Wir werden uns wiederfinden und ausgewählter Aktivitäten, die am Prinzip des handlungs- und produktorientierten Unterrichts orientiert sind, wollten wir zeigen, dass man mit 
literarischen Texten im DaF-Unterricht kreativ arbeiten kann und eine solche Form der Textanalyse auch ein Mittel sein kann, die Texte besser zu verstehen. Das an der Philosophischen Fakultät der Masaryk-Universität durchgeführte Projekt hat bestätigt, dass Emotionen bei einem Text eine sehr große Rolle spielen und ihre kreative Behandlung die ganze Persönlichkeit beeinflussen kann. Bei der Textanalyse wurde der Schwerpunkt auf die zwischenmenschlichen Beziehungen und ihre Darstellung im ausgewählten Buch gelegt.

Zusammenfassend lässt sich feststellen, dass die Unterrichtseinheiten geeignet ausgewählt wurden, dynamisch abliefen und den Studenten viele Impulse zu eigenem kreativen Handeln boten. Bei den Aktivitäten hatten sie die Möglichkeit, den Text zu analysieren und gleichzeitig ihre eigenen Meinungen und Vorstellungen zu präsentieren. Das Interesse der Studenten an der Arbeit war groß, was durch die Tatsache deutlich wurde, dass sie Interesse daran hatten, nicht nur einige Passagen, sondern das ganze Buch zu lesen. An diesem nicht traditionellen Unterrichtsverfahren waren die Studenten sehr interessiert und so wurden Misserfolge vermieden und alle Lernziele erreicht.

Abschließend ist zu bemerken, dass die Texte aus dem Bereich Kinder- und Jugendliteratur für den DaF-Unterricht geeignet sind, weil sie Interesse, Kreativität und Emotionen auf unterschiedlichsten Ebenen anzusprechen wissen.

\title{
Literaturverzeichnis
}

Fišer, Zbyněk (2012): Tvưrčípsaní v literárni výchově jako nástroj poznávání. Brno, Masarykova univerzita.

Grützmacher, Jutta (Hrsg.) (1979): Didaktik der Jugendliteratur. Analysen und Modelle für einen leserorientierten Deutschunterricht. Stuttgart, J.B.Metzlersche Verlagsbuchhandlung.

Haas, Gerhard (2001): Handlungs-und produktionsorientierter Literaturunterricht. Theorie und Praxis eines „anderen“ Literaturunterrichts für die Primär- und Sekundärstufe. 4. Auflage. Hannover, Kallmeyerche Verlagsbuchhandlung.

Haas, Gerhard / MENZEL, Wolfgang / SPINNER, Kaspar H. (1994): Handlungs- und produktionsorientierter Literaturunterricht. In: Praxis Deutsch. H.123. S. 17-25.

Kirsch, Dieter (2013): Szenisches Lernen: theaterarbeit im DaF-Unterricht. Ismaning, Hueber Verlag.

Kroll, Frank-Lothar (1997): Flucht und Vertreibung in der Literatur nach 1945. Berlin, Gebr. Mann Verlag.

Schwarz, Annelies (1988): Wir werden uns wiederfinden. 8. Auflage. München, Deutscher Taschenbuch Verlag.

Valenta, Josef (2008): Metody a techniky dramatické výchovy. Praha, Grada.

Wicke, Rainer E. (2004): Aktiv und kreativ lernen. Projektorientierte Spracharbeit im Unterricht. Ismaning, Hueber Verlag.

\author{
Milena Kubátová \\ Filozofická fakulta MU \\ Ústav germanistiky, nordistiky \\ a nederlandistiky
}

Brünner Hefte zu Deutsch als Fremdsprache • Jahrgang $7 \bullet$ Nummer 1/2 • 2014 